\title{
Protein negative/positively cooperative binding to zwitterionic/anionic vesicles
}

\author{
F Torrens ${ }^{*}, \mathrm{G}$ Castellano \\ From 5th German Conference on Cheminformatics: 23. CIC-Workshop \\ Goslar, Germany. 8-10 November 2009
}

The role of electrostatics is studied in the adsorption of cationic proteins to zwitterionic phosphatidylcholine (PC) and anionic mixed PC/phosphatidylglycerol (PG) small unilamellar vesicles (SUVs) [1]. For model proteins the interaction is monitored vs. PG content at low ionic strength [2]. The adsorption of lysozyme-myoglobin-bovine serum albumin (BSA) (isoelectric point, pI 5-11) is investigated in SUVs, along with changes of the fluorescence emission spectra of the proteins, via their adsorption on SUVs [3]. In the Gouy-Chapman formalism the activity coefficient goes with the square of charge number [4]. Deviations from the ideal model indicate asymmetric location of anionic phospholipid in the bilayer inner leaflet, in mixed zwitterionic/anionic SUVs for protein-PC/PG, in agreement with experiments-molecular dynamics simulations. Effective SUV charge stays constant. Myoglobin-, DNC-melittin- and melittin-zwitterionic associations are described by a partition model, modulated by electrostatic charging of membrane as protein accumulates at interface. Provisional conclusions follow. (1) In mixed zwitterionic/ anionic vesicles the charge effect on the protein binding model was analyzed. For lysozyme-anionic enough vesicles and myoglobin the electrostatic repulsion between cationic ad proteins dominates over the electrostatic attraction between ad protein dipoles. (2) The salt effect on the protein binding model of mixed zwitterionic/ anionic vesicles was analyzed. The cooperativity increases with ionic strength. The corresponding interpretation is that the electrostatic repulsion between cationic ad proteins decreases with increasing salt effect, and the electrostatic attraction between ad protein dipoles becomes dominant over the electrostatic repulsion between ad protein charges. (3) In anionic vesicles the effect of vesicle charge on protein binding shows that, with increasing anionic character of the vesicles, the protein-protein electrostatic repulsion is decreasingly important vs. the protein-vesicle attraction, and the electrostatic attraction between ad protein dipoles becomes dominant over the electrostatic repulsion between ad protein charges. (4) For lysozyme-mixed zwitterionic/ anionic vesicles and myoglobin cooperativity increases with $\mathrm{pH}$. With increasing $\mathrm{pH}$ and decreasing cationic character of the protein, the protein-protein electrostatic repulsion is decreasingly important against the proteinSUV attraction, and the electrostatic attraction between ad protein dipoles becomes dominant over the electrostatic repulsion between ad protein charges. Furthermore the opposed effect is observed for lysozyme-zwitterionic vesicles. (5) For protein-mixed zwitterionic/anionic vesicle binding there is more dispersion in the results, which could indicate asymmetric location of anionic phospholipid.

Published: 4 May 2010

\section{References}

1. Torrens F, Campos A, Abad C: Cell Mol Biol 2003, 49:991.

2. Torrens F, Abad C, Codoñer A, García-Lopera R, Campos A: Eur Polym J 2005, 41:1439.

3. Torrens F, Castellano G, Campos A, Abad C: J Mol Struct 2007, 216:834-836. 4. Torrens F, Castellano G, Campos A, Abad C: J Mol Struct 2009, 274:924-926.

doi:10.1186/1758-2946-2-S1-P12

Cite this article as: Torrens and Castellano: Protein negative/positively cooperative binding to zwitterionic/anionic vesicles. Journal of Cheminformatics 2010 2(Suppl 1):P12. 